\title{
Cell hybridization and cancer
}

\author{
J. F. WATKINS
}

From the Sir William Dunn School of Pathology, Oxford

In this paper I want to consider what, if anything, cell hybridization has contributed to our knowledge of malignant disease in animals, and whether it is possible that the technique would be useful in studies on human tumour cells. I shall deal almost exclusively with cell fusion produced by Sendai virus inactivated by ultraviolet light (Watkins, 1971). This virus, a parainfluenza virus related to mumps and Newcastle disease viruses, attaches to receptors on the cell membrane. If suspensions of two different kinds of cell are mixed with virus, clumps of cells are formed containing both cell types. Within the clumps cytoplasmic bridges form between some of the cells, and this leads to coalescence of their cytoplasms. If the suspension is allowed to settle on to coverslips in culture medium, Giemsa staining after overnight incubation shows multinucleated cells, many of which have nuclei of both parents, and are therefore called heterokaryons. On continued incubation the nuclei in some of the smaller heterokaryons enter mitosis synchronously, with the formation of a mixed metaphase which reconstitutes into a single nucleus to form a hybrid cell. The hybrid cell may continue to divide to form a hybrid clone, which can be separated from the parental cells by various selection procedures. During the development of the clone chromosomes are lost because of such phenomena as non-disjunction and failure of chromosomes to be incorporated at telophase. A hybrid clone is therefore genetically heterogeneous, and subclones can readily be selected from it by the application of suitable selection pressures. Another important property of heterokaryons and hybrid cells is that surface antigens of both parents are co-dominant, that is, are both expressed.

Cell fusion has been used in three areas of investigation of tumour cells: (1) attempts to recover virus from virus-transformed cells; (2) genetic analysis of malignancy; (3) tumour antigens.

\section{Virus Recovery from Virus-transformed Cells}

The principles of virus recovery, perhaps better called 'detection', can be illustrated by experiments on SV40 virus. When transformed mouse cells of a line known as SV3T3, which are nonpermissive for virus growth and from which no virus can be recovered by standard procedures, were fused with African Green Monkey kidney cells, about $5 \%$ of the heterokaryons formed produced SV40 virus. If the SV3T3 cells were treated with iododeoxyuridine (IUdR) for 24 hours before fusion the proportion of heterokaryons producing virus rose to over $80 \%$ (Watkins, 1970). This result is interesting in view of the subsequent demonstration by Lowy, Rowe, Teich, and Hartley (1971) that IUdR can induce certain transformed cell lines to produce C-type RNA viruses. The action of IUdR as a virus-inducing agent should be borne in mind in chemotherapy, since other agents which affect DNA synthesis may possibly have a similar effect. Recently I have extended these studies to a rabbit line transformed with SV40 virus. This line differs from SV3T3 in that about $0.03 \%$ of the cells are producing SV40 virus spontaneously. Cell fusion studies showed that about $3 \%$ of the heterokaryons produced SV40 virus, but the proportion could not be increased by treatment with iododeoxyuridine. In addition, hybridization in situ with heavily labelled RNA complementary to SV40 DNA showed that $2-3 \%$ of the cells were synthesizing viral DNA. The picture which begins to emerge from these and other studies suggests that SV40 virus may exist in a tumour cell line in one of two forms, possibly integrated and non-integrated. If the cell is capable of replicating the viral DNA it will do so when the virus is in the non-integrated form, but it will not go on to synthesize viral capsid protein until other conditions of an unknown kind are satisfied. Non-permissiveness may therefore be due to two blocks, in viral DNA synthesis and in viral protein synthesis. This model may be relevant to a consideration of the natural history of EB virus of Burkitt's lymphoma.

In contrast to SV40 virus, the related polyoma virus has not been recovered from transformed cells by fusion with permissive cells. Cell fusion is relevant to studies on C-type RNA viruses mainly in situations where the virus has transformed cells of a species other than its natural host. Svoboda and 
Dourmashkin (1969), for example, have recovered Rous sarcoma virus from transformed hamster cells by fusing them with chicken fibroblasts.

It will be obvious that the application of cell fusion to the search for a human tumour virus is not straightforward. First a cell line permissive for the putative virus must be found; this means that any human tumour must be screened against a wide range of cell types. Secondly, even if a permissive cell line could be found, the virus might behave like polyoma rather than SV40. If one speculated that a human tumour were caused by a C-type RNA virus from another species, for example, domestic cats or dogs, then, by analogy with Svoboda's studies on Rous virus, it might be worth using cells of the species in question in fusion studies.

\section{Genetic Analysis of Malignancy}

In the early 1960s Barski and Cornefert (1962) carried out some experiments to determine whether a hybrid line between a malignant cell and a nonmalignant cell would be malignant or nonmalignant. They concluded that the hybrid was malignant. Recently Klein and Harris (see Harris, 1971) and their colleagues re-investigated the question, using several different tumours of inbred mice crossed with a line of cells derived from $\mathrm{C} 3 \mathrm{H}$ mouse fibroblasts, and concluded that the hybrid was not malignant. They found, however, that the hybrid would revert to malignancy, and that the reversion was accompanied by a reduction in the number of chromosomes which had arisen from the normal parent. Two interpretations are possible. One is that reversion to malignancy occurred first, by some unknown genetic change, and the consequent rapid growth led to chromosome reduction; the other is that one or more chromosomes of the normal parent carry genes capable of suppressing malignancy, and reversion occurs because of random loss of certain chromosomes, among which may be chromosomes carrying suppressor genes. The second interpretation is supported by their finding that a hybrid formed between tumour cells and a malignant subclone of the original normal parent gave rise to a tumour in which there was little or no reduction in chromosome number in comparison with the population injected; that is, rapid growth, per se, of a hybrid tumour did not lead to gross chromosome loss. Future work with this system requires the demonstration that suppression of malignancy is associated with a particular chromosome or chromosomes but this is impossible in the absence of a method for selecting, in vivo, nonmalignant cells from malignant cells. There are no signs yet of such a method, which would, in itself, contribute greatly to the solution of the cancer problem.

As far as human tumours are concerned genetic analysis has no part to play unless interspecific hybrids can be made which turn out to be malignant: this would support the view that malignancy is a positive genetic character which may be associated with a specific chromosome. If Harris and Klein are right such a hybrid could not exist, even if problems of cross-species transplantation immunity were overcome.

\section{Tumour Antigens}

Finally, in the area of tumour antigens, one example of cell fusion studies must suffice to illustrate the kind of analysis which can be attempted. Mouse primary cells are not malignant when first transformed by SV40 virus, but malignant derivatives can sometimes be obtained on continued growth. This non-malignancy is usually attributed to the presence of SV40 transplantation antigens. However, Wesslén (1970) found that a malignant derivative of an SV40 transformed mouse line retained the SV40-specific transplantation antigen. I have been studying a hybrid population formed between a nonmalignant SV40-transformed $\mathrm{C} 3 \mathrm{H}$ mouse line and Ehrlich ascites tumour. The hybrid population is over $99 \%$ positive for SV40 T (intranuclear) antigen. The ascites tumours which arose in 14 out of 20 $\mathrm{C} 3 \mathrm{H}$ mice two weeks after intraperitoneal injection of $10^{6}$ hybrid cell had lower chromosome counts than the original culture, and 13 of the 14 tumours were over $99 \%$ negative for SV40 T antigen. Thus there seemed to be strong selection pressure in most of the mice against cells carrying SV40 virus: tumour cells which had presumably lost the SV40 genome grew faster in the peritoneal cavity than cells which had retained it. In fact, nearly all of the $10^{6}$ hybrid cells which were injected into each mouse must have been rejected. These results suggest either that the SV40 transplantation antigen is so strong for mice that it can even overcome the malignant potential of Ehrlich ascites tumour cells, which is at variance with Wesslén's result, or-and this would be extremely interesting - that SV40 transformed mouse cells are not a priori malignant, and, furthermore, the virus in the transformed cells may be linked with the genes which can apparently suppress the malignancy of Ehrlich ascites tumour cells. It is hoped that future experiments will enable a choice to be made between these two explanations.

As far as human tumours are concerned it should be possible, using the standard methods of somatic cell genetics, to assign tumour antigens to specific 
chromosomes. However, no experiments in this area have so far been reported.

\section{References}

Barski, G., and Cornefert, F. (1962). Characteristics of 'hybrid'type clonal cell lines obtained from mixed cultures in vitro. J. nat. Cancer Inst., 28, 801-822.

Harris, H. (1971). Cell fusion and the analysis of malignancy. Proc. roy. Soc. B., 179, 1-20.

Lowy, D. R., Rowe, W. P., Teich, N., and Hartley, J. W. (1971).
Murine leukemia virus: high-frequency activation in vitro by 5-iododeoxyuridine and 5-bromodeoxyuridine. Science, 174, 155-156.

Svoboda, J., and Dourmashkin, R. (1969). Rescue of Rous sarcoma virus from virogenic mammalian cells associated with chicken cells and treated with Sendai virus. J. gen. Virol., 4, 523-529.

Watkins, J. F. (1970). The effects of some metabolic inhibitors on the ability of SV40 virus in transformed cells to be detected by cell fusion. J. Cell Sci., 6, 721-737.

Watkins, J. F. (1971). Fusion of cells for virus studies and production of cell hybrids. Meth. Virol., 5, 1-32.

Wesslén, T. (1970). SV40-tumorigenesis in mouse. Acta path. microbiol. scand., Sec. B., 78, 479-487. 